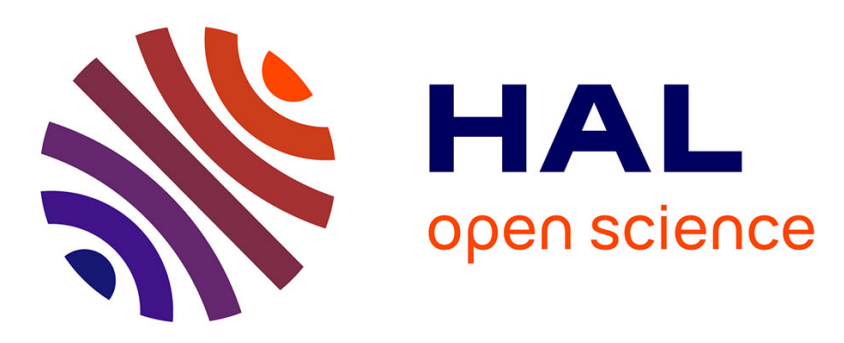

\title{
Linear time-invariant discrete delay systems in Laguerre domain
}

\author{
Alexander Medvedev, Viktor Bro, Rosane Ushirobira
}

\section{To cite this version:}

Alexander Medvedev, Viktor Bro, Rosane Ushirobira. Linear time-invariant discrete delay systems in Laguerre domain. IEEE Transactions on Automatic Control, 2021, 10.1109/TAC.2021.3096927 . hal-03323475

\section{HAL Id: hal-03323475 \\ https://hal.inria.fr/hal-03323475}

Submitted on 21 Aug 2021

HAL is a multi-disciplinary open access archive for the deposit and dissemination of scientific research documents, whether they are published or not. The documents may come from teaching and research institutions in France or abroad, or from public or private research centers.
L'archive ouverte pluridisciplinaire HAL, est destinée au dépôt et à la diffusion de documents scientifiques de niveau recherche, publiés ou non, émanant des établissements d'enseignement et de recherche français ou étrangers, des laboratoires publics ou privés. 


\title{
Linear time-invariant discrete delay systems in Laguerre domain
}

\author{
Alexander Medvedev, Member, IEEE, Viktor Bro, Student member, IEEE, and Rosane Ushirobira, Member, IEEE
}

\begin{abstract}
The paper provides the formulae connecting the Laguerre spectrum of the output signal of a linear discretetime time-invariant system to the Laguerre spectrum of its input signal and presents a standard state-space system description. The Laguerre domain system representation is meaningful when the input signal is square-summable and the system is stable. A certain type of polynomials arising in the evaluation of the output spectrum due to the presence of time delay is defined and key properties of these are investigated. The polynomials are characterized by a three-term recurrence relation that also facilitates their numerically reliable calculation.
\end{abstract}

Index Terms-Delay systems, linear systems, modeling.

\section{INTRODUCTION}

The problem of representing a signal as a point in a functional space lies in the heart of mathematical modeling. The coordinates of the point correspond then to the coefficients of a series approximating the signal. The ambition to keep the dimensionality of the functional space parsimonious immediately leads to the use of orthonormal functional bases and extensive tools have been developed in this classical field of mathematics during the last two centuries [1].

Since a linear time-invariant dynamical system is completely characterized by its impulse response, functional orthonormal bases can be equally applied to system modeling [2]. When a basis is spanned by functions with parameters, $a$ priori information about the system dynamics can be utilized for selecting values of the functional basis parameters so that the system behavior is captured with few terms, i.e. the resulting approximation is sparse.

Often, mathematical modeling is performed to design control or estimation algorithms by solving, generally speaking, an optimization problem. The numerical properties of the model are crucial for obtaining a robust design solution.

Model balancing involves an orthogonalization procedure and constitutes an effective way of improving numerical properties of state-space realizations as systems of difference or differential equations [3]. Yet other operators than difference and differential can be employed to capture the system dynamics. The quest for an alternative parametrization to improve numerical properties of models has resulted in e.g. $\delta$-operator [4] and $\gamma$-operator [5]. The former stems from the concept of

VB and AM were supported in part by the Swedish Research Council, under the grant 2019-04451.

VB and AM are with Information Technology, Uppsala University, Box 337, Uppsala SE-751 05, Sweden (e-mail: \{viktor.bro,alexander.medvedev\}@it.uu.se).

RU is with Inria, Univ. Lille, CNRS, UMR 9189 - CRIStAL, F-59000 Lille, France (e-mail: rosane.ushirobira@inria.fr) unifying the discrete- and continuous-time system frameworks, in particular by obtaining sampled-data models that converge to their continuous counterparts when the sampling time tends to zero. The latter operator has been originally introduced as a means of mitigating finite word length effects in digital implementation but has a wider field of potential applications.

As already mentioned, orthonormal functional bases with parameters offer a mathematically strict way of approaching the matter of favorable numerical properties in modeling. A sound choice for a basis representing linear time-invariant (LTI) systems in discrete and continuous time is the set of Laguerre functions [6] since they are orthogonalized polynomially weighted exponents [7], [8]. By mimicking the general form of a solution to an LTI system, Laguerre functions allow adjusting the effective time interval captured by the model through the value of a free parameter. The Laguerre series can be used to represent systems, as approximations of the transfer functions as operators, but also to describe input and output signals that decay at infinity. When the input-output signal pair is given in terms of Laguerre series, one speaks of a Laguerre domain system description.

The introduction of a continuous time-delay operator in a mathematical model results in an infinite-dimensional dynamics. Conventionally, time-delay systems are represented by differential-difference equations [9]. In the Laguerre domain, a time-delay system is given by a difference equation, but it is still infinite-dimensional [10]. In discrete time, a time delay increases the order of the state-space realization as well as imposes a specific block structure on the system matrix [9]. Since difference equations are inherently formulated in terms of the discrete-time shift operator, pure time delays in such a model are a matter of parametrization. Nevertheless, the numerical properties of a model are essential and tools for improving them are desired.

Continuous LTI systems with input or output delay were previously treated in Laguerre domain [10], [11]. The corresponding problem in discrete time has been addressed in full only recently [12]. Both in continuous and discrete time, certain types of polynomials arise in Laguerre domain description of time-delay systems. In continuous time, the polynomials are readily recognized as associated Laguerre polynomials [1], whereas the polynomials appearing in the discrete-time system description are not, to the best knowledge of the authors, covered in the literature and, therefore, are studied in the present contribution.

The main result of this paper is in formulating the distinctive properties of the polynomials arising in the Laguerre domain representation of discrete LTI systems with delay and 
completely characterizing the polynomial class at hand with a three-term relationship. A binomial identity that can also be of use in other areas (see Lemma 1) is proven as a partial step to the main result.

\section{THE DISCRETE LAGUERRE FUNCTIONS}

In the z-domain, the discrete Laguerre functions can be expressed as

$$
L_{j}(z)=\frac{\sqrt{1-p}}{z-\sqrt{p}} T^{j}(z), \quad T(z) \triangleq \frac{1-\sqrt{p} z}{z-\sqrt{p}},
$$

for all $j \in \mathbb{N}$, where the constant $0<p<1$ is the discrete Laguerre parameter.

Let $\mathbb{H}_{d}^{2}$ be the Hardy space of analytic functions on the complement of the unit disc, that are square-integrable on the unit circle. The functions $L_{j}(j \in \mathbb{N})$ constitute an orthonormal complete basis in $\mathbb{H}_{d}^{2}$ with respect to the inner product

$$
\langle W, V\rangle \triangleq \frac{1}{2 \pi i} \oint_{D} W(z) \overline{V(z)} \frac{\mathrm{d} z}{z},
$$

where $\overline{V(z)}=V\left(z^{-1}\right)$ and the contour integral is taken over the unit circle $D$.

From (1), the complete set of Laguerre functions can be obtained in terms of the discrete-time Laguerre shift operator $T$ by the recursion formula:

$$
L_{j+1}(z)=T(z) L_{j}(z), \forall j \in \mathbb{N} .
$$

Clearly, the orthogonality property of the Laguerre functions with respect to (2) is invariant to (forward or backward) time shift, so the basis

$$
L_{j}(z ; d)=z^{d} L_{0}(z) T^{j}(z), j=0,1, \ldots, d \in \mathbb{Z},
$$

is also orthonormal. This alternative definition (with $d=1$ ) is used for instance in [13].

Furthermore, the $j$-th Laguerre coefficient of $W \in \mathbb{H}_{d}^{2}$ is evaluated as the projection of $W$ onto $L_{j}$

$$
w_{j}=\left\langle W, L_{j}\right\rangle, \forall j \in \mathbb{N},
$$

and the set $\left\{w_{j}\right\}_{j \in \mathbb{N}}$ is referred to as the Laguerre spectrum of $W$. This parallels the notion of the Fourier spectrum that is obtained by projecting a function onto a set of harmonic functions, while the Laguerre spectrum is calculated by projecting it on a set of weighted (real) exponentials. Then, $W$ is completely defined by its Laguerre series or, in Laguerre domain, by the sequence of the Laguerre coefficients, i.e.

$$
W(z)=\sum_{k=0}^{\infty} w_{k} L_{k}, \quad W(z) \rightarrow\left[\begin{array}{llll}
w_{0} & w_{1} & w_{2} & \ldots
\end{array}\right] .
$$

The Laguerre shift operator $T$, defined in (1), acts as the right-shift operator $q$ on the sequence of Laguerre coefficients $\left\{w_{k}\right\}_{k \in \mathbb{N}}$, i.e. the Laguerre spectrum

$T(z) W(z)=\sum_{k=0}^{\infty} w_{k} T(z) L_{k}, \quad T(z) W(z) \rightarrow\left[\begin{array}{llll}0 & w_{0} & w_{1} & \ldots\end{array}\right]$,

or

$$
q\left[\begin{array}{llll}
w_{0} & w_{1} & w_{2} & \ldots
\end{array}\right]=\left[\begin{array}{llll}
0 & w_{0} & w_{1} & \ldots
\end{array}\right]
$$

The time domain representations of the Laguerre functions $\ell_{j}=z^{-1}\left\{L_{j}(z)\right\}(j \in \mathbb{N})$ yield an orthonormal basis in $\mathbb{1}_{2}=$ $\mathbb{1}_{2}[0, \infty)$, the space of square-summable sequences defined for non-negative arguments, where $z^{-1}$ denotes the inverse $z$ transform.

\section{LAGUERRE DOMAIN SYSTEM DESCRIPTION}

\section{A. Problem formulation}

Consider the LTI system with an input delay

$$
\begin{aligned}
x(t+1) & =A x(t)+B u(t-\tau), \forall t \in \mathbb{R}_{+} \\
y(t) & =C x(t),
\end{aligned}
$$

where $x: \mathbb{R} \rightarrow \mathbb{R}^{n}, A, B, C$ are real matrices of suitable dimensions, $x(0)=0$, and $\tau \in \mathbb{N}$. The problem at hand is to calculate the Laguerre spectrum of the output $\left\{y_{j}\right\}_{j \in \mathbb{N}}$ in terms of $A, B, C$, and $\tau$, given the Laguerre spectrum of the input $\left\{u_{j}\right\}_{j \in \mathbb{N}}$.

Proposition 1. Consider the discrete linear time-invariant system in (5), with $y, u \in \mathbb{1}_{2}$. For the input Laguerre spectrum $\left\{u_{j}\right\}_{j \in \mathbb{N}}$, the coefficients of the output Laguerre spectrum $\left\{y_{j}\right\}_{j \in \mathbb{N}}$ are given by

$$
\begin{aligned}
& y_{j}=(1-p) C(I-\sqrt{p} A)^{-2} \sum_{k=0}^{j-1} \overline{T(A)}{ }^{j-k-1} B v_{k}+ \\
& +\sqrt{p} C(I-\sqrt{p} A)^{-1} B v_{j},
\end{aligned}
$$

where

$$
\begin{gathered}
v_{j}=(1-p)(-1)^{j-\tau} \sum_{k=0}^{j-1} L_{j-k}^{(\tau)}(\sqrt{p}) u_{k}+\sqrt{p} \tau u_{j}, \\
L_{m}^{(\tau)}(\sqrt{p}) \triangleq(-\sqrt{p})^{m-\tau} \sum_{n=0}^{\tau-1}\left(\begin{array}{c}
m+n \\
n
\end{array}\right)\left(\begin{array}{c}
m-1 \\
\tau-n-1
\end{array}\right)(-p)^{n},
\end{gathered}
$$

and it is agreed that $\left(\begin{array}{l}n \\ k\end{array}\right)=0$ for $k>n$ by definition.

Proof. The proof is obtained by observing that (5) is composed of two blocks in series, one constituting an LTI system without explicit time delay and another that is a pure delay. The Laguerre-domain state-space equations for (5) are wellknown, see [14] and [13]. The Laguerre-domain description in (7) was recently obtained in [12].

The sum in (7) is a (discrete) convolution of the polynomials $L_{m}^{(\tau)}$ with the Laguerre spectrum of the input signal. In the convolution operation, each Laguerre coefficient of the input $u_{k}, k=0, \ldots, j-1$ is weighted by the polynomial $L_{j-k}^{(\tau)}$ and, therefore, all the input coefficients contribute to the value of $v_{j}$. This is in contrast with the finite memory of the delay operator in time domain.

Instead of projecting the input and output signal onto the polynomials $L_{j}$ as in (1), one can consider using the basis $L_{j}(z ; d)$ as defined in (3). Then the basis functions can be adapted to the properties of the modeled plant, i.e. (5), when there is a priori information about the delay value. Yet, when the plant (5) is subject to initial conditions, a delayed basis might have difficulty approximating the transient response in the output. Moreover, this approach is not feasible for systems with large delays. 


\section{COMPARISON TO CONTINUOUS CASE}

It is instructive to compare Laguerre-domain descriptions of the time delay operators in continuous and discrete time. A comparison between Laguerre-domain LTI models based on a unifying shift-operator description is provided in [13]. The Laplace transform of the $k$-th continuous Laguerre function is given by

$$
\ell_{k}(s)=\frac{\sqrt{2 p_{c}}}{s+p_{c}} T_{c}^{k}(s), \quad T_{c}(s) \triangleq \frac{s-p_{c}}{s+p_{c}},
$$

for $k \in \mathbb{N}$, where $p_{c}>0$ represents the Laguerre parameter, and $T_{c}$ is the continuous Laguerre shift operator.

Let $\mathbf{H}_{2}$ be the Hardy space of functions analytic in the open left half-plane. The set $\left\{\ell_{k}\right\}_{k \in \mathbb{N}}$ constitutes an orthonormal complete basis in $\mathbf{H}_{2}$ with respect to the inner product

$$
\langle W, V\rangle_{c} \triangleq \frac{1}{2 \pi i} \int_{-\infty}^{\infty} W(s) V(-s) \mathrm{d} s .
$$

The well-known family of associated Laguerre polynomials [1] is explicitly given by

$$
\mathrm{L}_{m}(\xi ; \alpha)=\sum_{n=0}^{m} \frac{1}{n !}\left(\begin{array}{c}
m+\alpha \\
m-n
\end{array}\right)(-\xi)^{n}, \forall m \in \mathbb{N}, \quad \xi \in \mathbb{R} .
$$

Denote $\mathrm{L}_{m}(\xi)=\left.\mathrm{L}_{m}(\xi ; \alpha)\right|_{\alpha=-1}$ and consider the continuous (pure) delay system

$$
y(t)=u\left(t-\tau_{c}\right), \tau_{c} \in \mathbb{R}_{+}, \forall t \in \mathbb{R}_{+} .
$$

For the system (10), the following relationship holds between the Laguerre coefficients of the input $\left\{u_{j}\right\}_{j \in \mathbb{N}}$ and those of the output $\left\{y_{j}\right\}_{j \in \mathbb{N}}$, all evaluated in sense of (8) [10], [11]:

$$
y_{j}=\mathrm{e}^{-\frac{\kappa}{2}}\left(\sum_{k=1}^{j} \mathrm{~L}_{k}(\kappa) u_{j-k}+u_{j}\right), \forall j \in \mathbb{N}, \text { where } \kappa=2 p \tau_{c} .
$$

For both (7) and (11), the Laguerre coefficients of the output are given by a discrete convolution between the Laguerre coefficients of the input and a polynomial kernel, where the number of terms in the convolution sum is equal to the Laguerre order of the output coefficient. Further, there is a direct term, i.e. $y_{j}$ that statically depends on $u_{j}$, this property is expected since the signals are related to each other as a whole and not their points or samples. Notably, the convolution operators are also "causal", in the sense that $u_{k}, k>j$ are not involved in right-hand side of (7) and (11).

There are also significant differences between (7) and (11). In continuous time, the argument of the kernel function incorporates a product of the Laguerre parameter $p_{c}$ and the delay value $\tau_{c}$, thus highlighting the time-scaling effect of $p_{c}$. In discrete time, the argument of the kernel function is the Laguerre parameter itself and the delay value is not involved. Therefore, the set of the kernel functions is completely defined and can be calculated once and for all feasible delays $\tau$ as soon as the value of the Laguerre parameter $p$ is specified.

Another principal difference is in the number of terms in the discrete and continuous case polynomials. In $L_{m}^{(\tau)}$, the number of terms is at most equal to the time delay, whereas the number of terms in $\mathrm{L}_{m}$ is defined by the order of the polynomial.
This difference is intimately related to the matter of delay operator dimensionality. The number of terms in the discrete case polynomial cannot exceed the time delay value since this is the dimension of the underlying dynamical system. In continuous time, the delay operator is infinite-dimensional and there is no limit to the number of terms in $\mathrm{L}_{m}$.

Notice that, both the continuous and discrete time, Laguerre shift operators are special cases of the symmetric bilinear transformation that is widely used in systems theory

$$
s \rightarrow \frac{a s+b}{c s-a}, \quad a^{2}+b c>0, \quad a, b, c \in \mathbb{R} .
$$

Bilinear transformations can be introduced formally, e.g. to improve numerical properties of a system identification algorithm [15] or with motivation from approximation theory and numerical integration, as for $\delta$-operator [4] and $\gamma$-operator [5] defined by

$$
\delta(z)=\frac{z-1}{T}, \quad \gamma(z)=\frac{2}{T} \frac{z-1}{z+1}, \quad T>0 .
$$

Obviously, $\gamma$-operator is closely related Tustin's discretization method

$$
z=\mathrm{e}^{T s}=\frac{1+T \frac{s}{2}}{1-T \frac{s}{2}}, \quad s=\frac{2}{T} \frac{1-z^{-1}}{1+z^{-1}},
$$

where $T$ is interpreted as the sampling time. An important observation that can be made from (6) is that the convolution kernel involves a matrix function of the state matrix $A$, namely

$$
\left.T(z)\right|_{z=A}=T(A) .
$$

Similarly, $T_{c}(A), \delta(A)$, and $\gamma(A)$ appear in the corresponding state-space representations, see [13], [4], [5].

\section{PROPERTIES OF THE POLYNOMIALS}

Some examples of the polynomials $L_{m}^{(\tau)}$ for different values of $\tau$ and $j$ are provided below for reference.

For $\tau=1$, the polynomial expression simplifies to

$$
L_{m}^{(1)}=(-\sqrt{p})^{m-1} .
$$

For $\tau=2$, the first five polynomials are

$$
\begin{aligned}
& L_{1}^{(2)}=2 \sqrt{p}, \quad L_{2}^{(2)}=1-3 \sqrt{p}^{2}, \quad L_{3}^{(2)}=-2 \sqrt{p}+4 \sqrt{p}^{3}, \\
& L_{4}^{(2)}=3 \sqrt{p}^{2}-5 \sqrt{p}^{4}, \quad L_{5}^{(2)}=-4 \sqrt{p}^{3}+6 \sqrt{p}^{5} .
\end{aligned}
$$

For $\tau=3$, the first five polynomials are

$L_{1}^{(3)}=3 \sqrt{p^{2}}, \quad L_{2}^{(3)}=3 \sqrt{p}-6 \sqrt{p}^{3}$, $L_{3}^{(3)}=1-8 \sqrt{p}^{2}+10 \sqrt{p}^{4}, L_{4}^{(3)}=-3 \sqrt{p}+15 \sqrt{p}^{3}-15 \sqrt{p}^{5}$, $L_{5}^{(3)}=6 \sqrt{p}^{2}-24 \sqrt{p}^{4}+21 \sqrt{p}^{6}$.

The polynomials $L_{m}^{(\tau)}$ possess interesting properties some of which can be established by inspection.

P1: All the powers in $L_{m}^{(\tau)}$ are either odd or even, which implies that the polynomials themselves are odd or even functions.

P2: There are at most $\tau$ terms in $L_{m}^{(\tau)}$. For $m \geq \tau$, all $L_{m}^{(\tau)}$ have exactly $\tau$ terms. 
P3: The lowest order of a term in $L_{m}^{(\tau)}$ is $|m-\tau|$, the highest is $\tau+m-2$. Therefore, only the polynomials $L_{\tau}^{(\tau)}$ have a free term.

Proposition 2. Let $S_{m}^{(\tau)}$ be the sum of the coefficients in the polynomial $L_{m}^{(\tau)}$, i.e.

$$
S_{m}^{(\tau)}=(-1)^{\tau-1} \sum_{n=0}^{\tau-1}(-1)^{n}\left(\begin{array}{c}
m+n \\
n
\end{array}\right)\left(\begin{array}{c}
m-1 \\
\tau-n-1
\end{array}\right) .
$$

Then $S_{m}^{(\tau)}=\tau$, for all $m \in \mathbb{N}$.

Proof. See Appendix A.

The polynomials $L_{m}^{(\tau)}$ can be obtained in a recursively.

Proposition 3. The following three-term recurrence relation

$$
L_{m+1}^{(\tau)}(\xi)=a_{m}^{(\tau)}(\xi) L_{m}^{(\tau)}(\xi)+b_{m} L_{m-1}^{(\tau)}(\xi)
$$

holds with

$$
\begin{gathered}
a_{m}^{(\tau)}(\xi) \triangleq a_{m, 1}^{(\tau)} \xi+a_{m, 2}^{(\tau)} \xi^{-1} \\
a_{m, 1}^{(\tau)}=-\frac{m+\tau}{m+1}, \quad a_{m, 2}^{(\tau)}=-\frac{m-\tau}{m+1}, \quad b_{m}=-\frac{m-1}{m+1} .
\end{gathered}
$$

Proof. See Appendix B.

For $\tau=2$

$$
\begin{aligned}
& a_{2}^{(2)}(\xi)=-\frac{4}{3} \xi, \quad b_{2}=-\frac{1}{3} \\
& a_{3}^{(2)}(\xi)=-\frac{5}{4} \xi-\frac{1}{4} \xi^{-1}, \quad b_{3}=-\frac{1}{2} \\
& a_{4}^{(2)}(\xi)=-\frac{6}{5} \xi-\frac{2}{5} \xi^{-1}, \quad b_{4}=-\frac{3}{5}
\end{aligned}
$$

For $\tau=3$

$$
\begin{aligned}
& a_{2}^{(3)}(\xi)=-\frac{5}{3} \xi+\frac{1}{3} \xi^{-1}, \quad b_{2}=-\frac{1}{3} \\
& a_{3}^{(3)}(\xi)=-\frac{3}{2} \xi, \quad b_{3}=-\frac{1}{2} \\
& a_{4}^{(3)}(\xi)=-\frac{7}{5} \xi-\frac{1}{5} \xi^{-1}, \quad b_{4}=-\frac{3}{5}
\end{aligned}
$$

Notice that Favard's theorem securing orthogonality of a polynomial set through a three-term recurrence relation demands that

$$
a_{m}^{(\tau)}(\xi)=\xi+c_{m}
$$

for some $c_{m}$, is satisfied. While the matter of $a_{m}^{(\tau)}$ being monic can be settled through normalization, yet (13) cannot be reduced to a first-order polynomial. Therefore, the orthogonality of $L_{m}^{(\tau)}$ remains an open question.

In continuous time, the associated Laguerre polynomials obey the following three-term relationship

$$
\mathrm{L}_{m+1}(\xi)=\frac{1}{m+1}(\xi+2 m) \mathrm{L}_{m}(\xi)-\frac{m-1}{m+1} \mathrm{~L}_{m-1}(\xi),
$$

and the orthogonality follows immediately.

The presence of $\xi^{-1}$ in (13) is a consequence of $\mathbf{P 2}$ since the lower powers of $\xi$ have to be canceled when the order of $L_{m}^{(\tau)}(\xi)$ is increased. This follows from that fact that the discrete-time delay operator has finite dimension equal to $\tau$. In the continuous-time polynomials $\mathrm{L}_{m}(\xi)$, the number of terms grows with the polynomial order.

\section{DISCUSSION}

A system with a time delay in the input or the output signal constitutes a cascade coupling of two dynamical subsystems with dissimilar properties. When both subsystems are unknown and must be identified from input-output data, the parameter estimation problem becomes challenging [16].

Transforming a continuous LTI delay system into the Laguerre domain results in an equivalent discrete state-space description of the dynamics without resorting to a sampling procedure. Besides, the model acquires an extra degree of freedom, the Laguerre parameter, that can be utilized to obtain beneficial numerical properties. In the Laguerre domain, the continuous delay operator turns into the discrete convolution operator in (10). Since the convolution kernel is polynomial and depends on the unknown delay value, the system identification problem becomes nonlinear. Despite the deceivingly simple discrete form, the operator is still infinite-dimensional and cannot be expressed in a state-space form with a finite number of states.

In discrete time, the effect of transforming an LTI delay system into the Laguerre domain is twofold. On the one hand, similarly to continuous time, the model can be regularized by employing the Laguerre parameter. On the other hand, the convolution operator in (7) does not include the delay value in the argument of the kernel but rather, as the sharp bound on the number of the terms in the kernel polynomials. The convolution operator is finite-dimensional, as follows from the finite number of the polynomial terms, but of unknown order since the time delay is unknown.

In the system identification context, a practical (but probably not the best) way of identifying time-delay systems in the Laguerre domain is gridding over an interval of feasible time delay values and solving an LTI identification problem for each point of the grid. Indeed, since time delay is a non-negative and bounded parameter, as well as the interval of suitable values is usually a priori known in biomedical applications, this approach is demonstrated to produce good results [10], [12].

Laguerre function basis has for a long time been used in nonlinear modeling to parametrize kernels in Volterra (convolution) models [17], thus leading to so-called VolterraLaguerre (VL) models. The structure of a Volterra model readily allows for a time delay in the input or the output signal, albeit implicitly. When an estimate of the delay is sought, the parameter has to appear explicitly in the model. This is achieved by attributing the delay to the kernels of the Volterra model and projecting them onto the Laguerre basis. Assuming the kernels are separable and approximating them by truncated Laguerre series conveniently justifies their interpretation as impulse responses of LTI systems, cf. (5). Continuous VL models with explicit time delay are dealt with in [18] and the discrete counterpart is covered in [19], applied to mathematical modeling of the human smooth pursuit system.

\section{CONCLUSION}

Laguerre domain descriptions of dynamical systems provide a common structure for modeling of continuous and discrete 
systems along with enforcing beneficial numerical properties of the models. The utility of this framework for time-delay systems was highlighted and mathematical properties of the resulting models were analyzed, in particular with respect to the special types of polynomials arising in mapping system descriptions from the time domain to the Laguerre domain.

\section{REFERENCES}

[1] G. Szegő, Orthogonal Polynomials. American Mathematical Society, 1939, colloquium Publications. XXIII.

[2] P. S. Heuberger, P. M. van den Hof, and B. Wahlberg, Modelling and Identification with Rational Orthogonal Basis Functions. Springer, 2005.

[3] A. J. Laub, M. T. Heath, C. C. Paige, and R. C. Ward, "Computation of system balancing transformations - and other applications of simultaneous diagonalization algorithms," IEEE Transactions on Automatic Control, vol. 32, no. 2, pp. 115-122, February 1987.

[4] R. H. Middleton and G. C. Goodwin, Digital Control and Estimation: A Unifying Approach. Englewood Cliffs, NJ: Prentice Hall, 1990.

[5] M. Gevers and G. Li, Parametrizations in Control, Estimation and Filtering Problems: Accuracy Aspects. London: Springer, 1993.

[6] P. R. Clement, "Laguerre functions in signal analysis and parameter identification," Journal of the Franklin Institute, vol. 313, no. 2, pp. 85-95, February 1982.

[7] B. Wahlberg, "System identification using Laguerre models," IEEE Transactions on Automatic Control, vol. 36, no. 5, pp. 551-562, 91.

[8] P. Mäkilä and J. Partington, "Laguerre and Kautz shift approximations of delay systems," Int. J. Control, vol. 72, no. 10, pp. 932-946, 1999.

[9] E. Fridman, "Discrete-time delay systems," in Introduction to TimeDelay Systems. Systems \& Control: Foundations \& Applications. Cham: Birkhäuser, 2004.

[10] E. Hidayat and A. Medvedev, "Laguerre domain identification of continuous linear time delay systems from impulse response data," Automatica, vol. 48, no. 11, pp. 2902-2907, 2012.

[11] B. Fischer and A. Medvedev, " $L_{2}$ time delay estimation by means of Laguerre functions," in Proceedings of the 1999 American Control Conference, San Diego, CA, 1999.

[12] V. Bro, A. Medvedev, and R. Ushirobira, "Laguerre-domain modelling and identification of linear discrete-time delay systems," in IFAC World Congress, Berlin, Germany, July 2020, accepted.

[13] B. Fischer and A. Medvedev, "Laguerre shift identification of a pressurized process," in Proceedings of the 1998 American Control Conference, vol. 3, 1998, pp. 1933-1937.

[14] Y. Nurges and Y. Yaaksoo, "Laguerre state equations for multivariable discrete systems," Autom. Rem. Control, vol. 42, pp. 1601-1603, 1982.

[15] M. Verhaegen, D. Westwick, and R. Kearney, "The use of a bilinear transformation of the shift operator in subspace model identification," IEEE Transactions on Automatic Control, vol. 40, no. 8, pp. 1422-1428, 1995.

[16] L. Belkoura, J.-P. Richard, and M. Fliess, "Parameters estimation of systems with delayed and structured entries," Automatica, vol. 45, pp. $1117-1125,2009$.

[17] N. Wiener, "Nonlinear problems in random theory," Nonlinear Problems in Random Theory, by Norbert Wiener, pp. 142. ISBN 0-262-73012-X. Cambridge, Massachusetts, USA: The MIT Press, August 1966.(Paper), p. 142, 1966.

[18] V. Bro and A. Medvedev, "Identification of continuous Volterra models with explicit time delay through series of Laguerre functions," in IEEE Conference on Decision and Control, Nice, France, 2019.

[19] _ - "Continuous and discrete Volterra-Laguerre models with delay for modeling of smooth pursuit eye movements," in IEEE Conference on Decision and Control, Jeju Island, Korea, 2020, submitted.

[20] S. M. Ruiz, "An algebraic identity leading to Wilson's theorem," The Mathematical Gazette, vol. 80, no. 489, pp. 579-582, November 1996.

\section{APPENDIX A}

\section{ProOF OF PROPOSITION 2}

To prove the sought result, an auxiliary lemma is required.

Lemma 1. The following identity holds for all $m, \tau \in \mathbb{N}$

$$
(-1)^{\tau} \sum_{n=0}^{\tau}(-1)^{n}\left(\begin{array}{c}
m+n \\
n
\end{array}\right)\left(\begin{array}{c}
m \\
\tau-n
\end{array}\right)=1
$$

Proof. First, notice that the terms in the sum in (15) are all polynomials in $m$ of order $\tau$. Using the factorial formula for the binomial coefficients gives

$$
\begin{aligned}
& \left(\begin{array}{c}
m+n \\
n
\end{array}\right)\left(\begin{array}{c}
m \\
\tau-n
\end{array}\right)=\frac{(m+n) \tau}{n !(\tau-n) !}= \\
& \frac{1}{n !(\tau-n) !} \sum_{k=0}^{\tau} s(\tau, k)(m+n)^{k},
\end{aligned}
$$

where $s(\cdot, \cdot)$ are the Stirling numbers of the first kind and $(x)_{n}$ is the falling factorial, i.e.

$$
(m+n)_{\tau}=(m+n)(m+n-1) \ldots(m+n-\tau+1) .
$$

Then, for the result to hold, the sum of the coefficients by each non-zero power of $m$ in (15) should be zero, and the free term has to be equal to one. The latter property is checked by calculating

$$
m=0: \quad(-1)^{\tau} \sum_{n=0}^{\tau}(-1)^{n}\left(\begin{array}{l}
n \\
n
\end{array}\right)\left(\begin{array}{c}
0 \\
\tau-n
\end{array}\right)=(-1)^{2 \tau}=1 .
$$

The leading coefficient (by $m^{\tau}$ )

$$
\begin{aligned}
& (-1)^{\tau} \sum_{k=0}^{\tau} \sum_{n=0}^{\tau} \frac{(-1)^{n}}{n !(\tau-n) !} s(\tau, k)=(-1)^{\tau} \sum_{k=0}^{\tau} s(\tau, k) \sum_{n=0}^{\tau} \frac{(-1)^{n}}{n !(\tau-n) !}= \\
& \frac{(-1)^{\tau}}{\tau !} \sum_{k=0}^{\tau} s(\tau, k) \sum_{n=0}^{\tau}(-1)^{n}\left(\begin{array}{l}
\tau \\
n
\end{array}\right)=0,
\end{aligned}
$$

in virtue of the identity

$$
\sum_{n=0}^{\tau}(-1)^{n}\left(\begin{array}{l}
\tau \\
n
\end{array}\right)=0
$$

The sum of the coefficients by a certain power of $m$ can be obtained by derivation of the left-hand side of (15) with respect to $m$

$$
\begin{gathered}
\frac{\mathrm{d}^{j}}{\mathrm{~d} m^{j}}(-1)^{\tau} \sum_{n=0}^{\tau} \frac{(-1)^{n}}{n !(\tau-n) !} \sum_{k=0}^{\tau} s(\tau, k)(m+n)^{k}= \\
(-1)^{\tau} \sum_{n=0}^{\tau} \frac{(-1)^{n}}{n !(\tau-n) !} \sum_{k=0}^{\tau} s(\tau, k) \frac{\mathrm{d}^{j}}{\mathrm{~d} m^{j}}(m+n)^{k}= \\
(-1)^{\tau} \sum_{n=0}^{\tau} \frac{(-1)^{n}}{n !(\tau-n) !} \sum_{k=0}^{\tau} s(\tau, k)(k)_{j}(m+n)^{k-j}= \\
\frac{(-1)^{\tau}}{\tau !} \sum_{k=0}^{\tau} s(\tau, k)(k)_{j} \sum_{n=0}^{\tau}(-1)^{n}\left(\begin{array}{c}
\tau \\
n
\end{array}\right)(m+n)^{k-j} .
\end{gathered}
$$

Now, from Corollary 2 in [20], for $1 \leq j \leq \tau$

$$
\sum_{n=0}^{\tau}(-1)^{n}\left(\begin{array}{l}
\tau \\
n
\end{array}\right)(m+n)^{k-j}=0
$$

which completes the proof.

Now the claim of Proposition 2 can be proven.

Proof. The result is proven by induction with respect to $\tau$. For $\tau=1$, it is easily checked that

$$
S_{m}^{(1)}=(-1)^{0}\left(\begin{array}{c}
m \\
0
\end{array}\right)\left(\begin{array}{c}
m-1 \\
0
\end{array}\right)=1, \quad \forall m .
$$


Since $m$ does not actually appear in the previous expression, it is instructive to evaluate

$$
\begin{aligned}
& S_{m}^{(2)}=(-1)^{1}\left(\begin{array}{c}
m \\
0
\end{array}\right)\left(\begin{array}{c}
m-1 \\
1
\end{array}\right)+(-1)^{0}\left(\begin{array}{c}
m+1 \\
1
\end{array}\right)\left(\begin{array}{c}
m-1 \\
0
\end{array}\right) \\
& =-(m-1)+(m+1)=2, \quad \forall m,
\end{aligned}
$$

to reveal the mechanism by which the powers of $m$ cancel each other.

Now consider

$$
S_{m}^{(\tau+1)}=(-1)^{\tau} \sum_{n=0}^{\tau}(-1)^{n}\left(\begin{array}{c}
m+n \\
n
\end{array}\right)\left(\begin{array}{c}
m-1 \\
\tau-n-1
\end{array}\right) .
$$

By using the identity

$$
\left(\begin{array}{c}
m-1 \\
\tau-n
\end{array}\right)=\left(\begin{array}{c}
m \\
\tau-n
\end{array}\right)-\left(\begin{array}{c}
m-1 \\
\tau-n-1
\end{array}\right)
$$

one has

$$
\begin{aligned}
S_{m}^{(\tau+1)} & =(-1)^{\tau} \sum_{n=0}^{\tau}(-1)^{n}\left(\begin{array}{c}
m+n \\
n
\end{array}\right)\left(\begin{array}{c}
m \\
\tau-n
\end{array}\right) \\
& +(-1)^{\tau-1} \sum_{n=0}^{\tau-1}(-1)^{n}\left(\begin{array}{c}
m+n \\
n
\end{array}\right)\left(\begin{array}{c}
m-1 \\
\tau-n-1
\end{array}\right) .
\end{aligned}
$$

Finally,

$$
S_{m}^{(\tau+1)}=S_{m}^{(\tau)}+(-1)^{\tau} \sum_{n=0}^{\tau}(-1)^{n}
$$

where the last identity is due to Lemma 1.

\section{APPENDIX B \\ PROOF OF PROPOSITION 3}

According to $\mathbf{P 2}$, the polynomials $L_{m}^{(\tau)}$ cannot have more than $\tau$ terms for any $m$. The polynomials $L_{m+1}^{(\tau)}$ and $\xi L_{m}^{(\tau)}$ comprise terms of power from $m-\tau+1$ to $m+\tau-1$, while the terms in the polynomials $L_{m-1}^{(\tau)}$ and $\xi^{-1} L_{m}^{(\tau)}$ are of power from $m-\tau-1$ to $m+\tau-3$.

The leading coefficient of $L_{m+1}^{(\tau)}$ (the coefficient of $\xi^{m-\tau+1}$ ) coincides with that of $\xi a_{m, 1}^{(\tau)} L_{m}^{(\tau)}$ since

$$
\frac{m+\tau}{\tau-1}\left(\begin{array}{c}
m+\tau-1 \\
\tau-1
\end{array}\right)\left(\begin{array}{c}
m-1 \\
0
\end{array}\right)=\left(\begin{array}{c}
m+\tau \\
\tau-1
\end{array}\right) .
$$

The next coefficient (multiplying $\xi^{m-\tau}$ ) is zero in both polynomials because of P1. Further, for recursion (12) to hold, the least power term of $a_{m, 2}^{(\tau)} \xi^{-1} L_{m}^{(\tau)}$ must cancel the least power term in $b_{m} L_{m-1}^{(\tau)}$ which follows since

$$
\frac{m-\tau}{m+1}\left(\begin{array}{c}
m \\
0
\end{array}\right)\left(\begin{array}{c}
m-1 \\
\tau-1
\end{array}\right)-\frac{m-1}{m+1}\left(\begin{array}{c}
m-1 \\
0
\end{array}\right)\left(\begin{array}{c}
m-2 \\
\tau-1
\end{array}\right) \equiv 0 .
$$

Once again, the coefficients multiplying $\xi^{m+\tau-2}$ are zero, both in $\xi^{-1} L_{m}^{(\tau)}$ and $b_{m} L_{m-1}^{(\tau)}$, due to $\mathbf{P 1}$.

Now, it has to be shown that the relation

$$
\left(\begin{array}{c}
m+k+1 \\
k
\end{array}\right)\left(\begin{array}{c}
m \\
\tau-1-k
\end{array}\right)(-1)^{m-\tau+k}=s_{1}(k)+s_{2}(k)+s_{3}(k),
$$

where

$$
\begin{aligned}
& s_{1}(k)=-\frac{m+\tau}{m+1}\left(\begin{array}{c}
m+k \\
k
\end{array}\right)\left(\begin{array}{c}
m-1 \\
\tau-1-k
\end{array}\right)(-1)^{m-\tau+k}, \\
& s_{2}(k)=-\frac{m-\tau}{m+1}\left(\begin{array}{c}
m+k+1 \\
k+1
\end{array}\right)\left(\begin{array}{c}
m-1 \\
\tau-2-k
\end{array}\right)(-1)^{m-\tau+k+1}, \\
& s_{3}(k)=-\frac{m-1}{m+1}\left(\begin{array}{c}
m+k \\
k+1
\end{array}\right)\left(\begin{array}{c}
m-2 \\
\tau-2-k
\end{array}\right)(-1)^{m-\tau+k},
\end{aligned}
$$

holds for $k=0, \ldots, \tau-2$ (i.e. the powers $m-\tau+1$ to $m+\tau-$ 3 ), where the non-zero coefficients of all three polynomials $\xi L_{m}^{(\tau)}, \xi^{-1} L_{m}^{(\tau)}$ and $L_{m-1}^{(\tau)}$ are involved in the right-hand side of (12).

Consider first the sum

$$
\begin{aligned}
s_{1}(k)+s_{3}(k) & =\frac{(-1)^{m-\tau+k+1}}{m+1}\left(\left(\begin{array}{c}
m+k \\
k
\end{array}\right)\left(\begin{array}{c}
m-1 \\
\tau-1-k
\end{array}\right)(m+\tau)\right. \\
& \left.+(m-1)\left(\begin{array}{c}
m+k \\
k+1
\end{array}\right)\left(\begin{array}{c}
m-2 \\
\tau-2-k
\end{array}\right)\right) .
\end{aligned}
$$

By applying the identities

$$
\left(\begin{array}{c}
m-1 \\
\tau-1-k
\end{array}\right)=\frac{m-1}{\tau-1-k}\left(\begin{array}{c}
m-2 \\
\tau-2-k
\end{array}\right), \quad\left(\begin{array}{c}
m+k \\
k+1
\end{array}\right)=\frac{m}{k+1}\left(\begin{array}{c}
m+k \\
k
\end{array}\right),
$$

it can be concluded that

$s_{1}(k)+s_{3}(k)=\frac{(-1)^{m-\tau+k+1}}{m(m+1)}\left(\begin{array}{c}m-1 \\ \tau-1-k\end{array}\right)\left(\begin{array}{c}m+k \\ k+1\end{array}\right) \tau(\tau+m+1)$.

Now consider the whole right-hand side expression in (12)

$$
\begin{aligned}
& s_{1}(k)+s_{2}(k)+s_{3}(k)= \\
& \frac{(-1)^{m-\tau+k+1}}{m+1}\left(\left(\begin{array}{c}
m-1 \\
\tau-1-k
\end{array}\right)\left(\begin{array}{c}
m+k \\
k+1
\end{array}\right) \frac{\tau(k+m+1)}{m}\right. \\
& \left.-(m-\tau)\left(\begin{array}{c}
m+k+1 \\
k+1
\end{array}\right)\left(\begin{array}{c}
m-1 \\
\tau-2-k
\end{array}\right)\right) .
\end{aligned}
$$

Making use of the identities

$$
\begin{aligned}
& \left(\begin{array}{c}
m-1 \\
\tau-k-1
\end{array}\right)=\frac{m-\tau+k+1}{\tau-k-1}\left(\begin{array}{c}
m-1 \\
\tau-k-2
\end{array}\right), \\
& \left(\begin{array}{c}
m+k+1 \\
k+1
\end{array}\right)=\frac{m+k+1}{m}\left(\begin{array}{c}
m+k \\
k+1
\end{array}\right),
\end{aligned}
$$

one arrives to

$$
\begin{aligned}
& s_{1}(k)+s_{2}(k)+s_{3}(k)= \\
& \frac{(-1)^{m-\tau+k+1} m(k+1)}{(m+1)(m-\tau+k+1)}\left(\begin{array}{c}
m-1 \\
\tau-1-k
\end{array}\right)\left(\begin{array}{c}
m+k+1 \\
k+1
\end{array}\right) .
\end{aligned}
$$

Finally, by observing that

$$
\begin{aligned}
& \left(\begin{array}{c}
m \\
\tau-k-1
\end{array}\right)=\frac{m}{m-\tau+k+1}\left(\begin{array}{c}
m-1 \\
\tau-k-1
\end{array}\right), \\
& \left(\begin{array}{c}
m+k+1 \\
k+1
\end{array}\right)=\frac{m+1}{k+1}\left(\begin{array}{c}
m+k+1 \\
k
\end{array}\right),
\end{aligned}
$$

the equality in (16) is proven. 